\title{
Different applications of RRE on nonsymmetric algebraic Riccati equation in transport theory
}

\author{
Rola El Moallem ${ }^{1, *}$, Hassane Sadok ${ }^{2}$ \\ ${ }^{1}$ College of Science and Information Systems, Rafik Hariri University, 10 Damour-Chouf, Lebanon \\ University Institute of Technology, Lebanese University, Lebanon \\ ${ }^{2}$ Laboratoire de Mathématiques Pure et Appliquées, Université du Littoral, Centre Universitaire de la \\ Mi-Voix, Batiment H. Poincarré, 50 Rue F. Buisson, BP 699, 62228 Calais Cedex, France
}

\begin{abstract}
This paper proposes three different ways of applying a vector extrapolation method to a slow converging vector sequence to accelerate the convergence. The solution of this vector sequence is used to solve an algebraic Riccati equation in transport theory. A numerical example is presented to compare the three applications.
\end{abstract}

\section{Introduction}

The nuclear disaster that occurred in japan on March 2011 emphasizes the vulnerability of the population at the vicinity of the nuclear power plant. Nonsymmetric algebraic Riccati equations arise in many fields such as transport theory when dealing with particle transfer (or radiative transfer), nuclear physics, control theory, etc. In this paper, we are interested in special Riccati equations that arise in transport theory. An important problem is computing limits of high dimension vector sequences. Many of these sequences converge extremely slowly to their limits. Such vector sequences may arise for example from finite-difference or finite-element discretizations of continuum problems. In such problems, as the mesh sizes decrease, their speed of convergence gets worse. Other vector sequences may result from iterative solution of linear or nonlinear systems of equations. This requires the use of convergence acceleration methods.

Vector extrapolation methods may be applied to accelerate the convergence of vector sequences. These techniques transform a sequence of vectors generated by some process to a new one that converges faster than the initial sequence.

\section{Nonsymmetric algebraic Riccati equation}

Riccati equations are matrix equations with a quadratic term. These equations were first examined by Jacopo Francesco Riccati in 1723 and still being examined till nowadays. A matrix version of such equations was introduced much later.

\footnotetext{
* Corresponding author: moallemrf@rhu.edu.lb
} 
These equations arise in continuous-time and discrete-time linear-quadratic-Gaussian control. Algebraic Riccati equations denote the non-dynamic version of Riccati equations.

This paper is interested in a nonsymmetric algebraic Riccati equation (NARE) of the form

$$
X A X-X D-A X+B=0
$$

with a real square unknown matrix $\mathrm{X}$ and real matrices, $\mathrm{A}, \mathrm{B}, \mathrm{C}$ and $\mathrm{D} \in \mathbb{R}^{\mathrm{n} \times \mathrm{n}}$ as matrix coefficients.

Nonsymmetric algebraic Riccati equations(NARE) arise in different fields such as transport theory, engineering, nuclear physics, control theory, applied probability, and much more. These equations play a crucial role in two important applications, namely, the analysis of transport equations [1,2] and the study of fluid queues models [3,4,5].

This paper deals with a nonsymmetric algebraic Riccati equations which occurs in transport theory with specific values of matrix coefficients, that is the one which concerns particle transport or radiative transfer.

Here, the minimal positive solution $\mathrm{X}^{*}$, that is the one with componentwise minimal entries, is the solution of interest and of physical meaning. In [6], it was seen that the minimal positive solution of a certain vector equation leads to the minimal positive solution of (NARE). Using the method in [7] and the modified version in [8], the minimal positive solution of NARE can be computed as follows

$$
X=T \circ\left(u v^{T}\right),
$$

where ${ }^{\circ}$ denotes the Hadamard product, $u$ and $v \in \mathbb{R}^{\mathrm{n} \times 1}$, and $\mathrm{T} \in \mathbb{R}^{\mathrm{n} \times \mathrm{n}}$ is given.

Then, finding two positive vector $\mathrm{u}$ and $\mathrm{v}$ leads to the minimal positive solution of (1). These vectors can be computed as in [8] by the following iteration

$$
w^{(k+1)}=\left[\begin{array}{l}
u^{k+1} \\
v^{k+1}
\end{array}\right]:=\left[\begin{array}{c}
\left(I_{n}-\operatorname{diag}\left(\mathrm{P} v^{(k)}\right)\right)^{-1} \mathrm{e} \\
\left(I_{n}-\operatorname{diag}\left(\mathrm{Q} u^{(k+1)}\right)\right)^{-1} \mathrm{e}
\end{array}\right]
$$

where $\mathrm{P}$ and $\mathrm{Q}$ are given and $\mathrm{k}=0,1, \ldots$

Convergence of vector sequences tend to be very slow, then we will apply a vector extrapolation technique, namely the reduced rank exrapolation (RRE) to accelerate the convergence.

\section{Reduced rank exrapolation}

The reduced rank exrapolation technique was introduced by Eddy [9] and Mesina [10]. An advantage of ths technique is that it does not require an explicit knowledge of the way the sequence is generated. Also it can be applied to the solution of linear and nonlinear systems of equations.

Given $\left\{\mathrm{u}^{(\mathrm{k})}\right\}_{\mathrm{k} \in \mathrm{N}}$ a sequence of vectors in $\mathbb{R}^{\mathrm{n}}$. Define the first and second forward differences of $u^{(k)}$ by

$$
\Delta u^{(k)}=u^{(k+1)}-u^{(k)} \quad \text { and } \quad \Delta^{2} u^{(k)}=\Delta u^{(k+1)}-\Delta u^{(k)}, \quad k=0,1, \ldots
$$

When RRE is applied to the vector sequence $\left\{\mathrm{u}^{(\mathrm{k})}\right\}_{\mathrm{k} \in \mathrm{N}}$, this technique produces an approximation $\mathrm{s}^{(\mathrm{k})}$ of the limit of $\left\{\mathrm{u}^{(\mathrm{k})}\right\}_{\mathrm{k} \in \mathrm{N}}$.

The approximation $\mathrm{s}^{(\mathrm{k})}$ is defined by 


$$
s^{(k)}=\sum_{j=0}^{k} \beta_{j} u^{(j)},
$$

where $\sum_{j=0}^{k} \beta_{j}=1$ and $\sum_{j=0}^{k} \gamma_{i, j} \beta_{j}=0$, for $i=0,1, \ldots, k-1$.

The scalars $\gamma_{i, j} \in \mathbb{R}$ are defined for $\mathrm{i}=0,1, \ldots, \mathrm{k}-1$ and $\mathrm{j}=0,1, \ldots, \mathrm{k}$ by

$$
\gamma_{i, j}=\left(\Delta^{2} u^{(i)}, \Delta u^{(j)}\right) .
$$

Using the expression $\Delta^{i} U^{(k)}=\left[\Delta^{i} u^{(0)}, \ldots, \Delta^{i} u^{(k-1)}\right]$ and Schur complements, $\mathrm{s}^{(\mathrm{k})}$ can be written as

$$
s^{(k)}=u^{(0)}-\Delta U^{(k)}\left(\Delta^{2} U^{(k)}\right)^{+} \Delta u^{(0)},
$$

where $\left(\Delta^{2} U^{(k)}\right)^{+}$denotes the Moore-Penrose generalized inverse of $\Delta^{2} U^{(k)}$ defined by

$$
\left(\Delta^{2} U^{(k)}\right)^{+}=\left(\left(\Delta^{2} U^{(k)}\right)^{T} \Delta^{2} U^{(k)}\right)^{-1}\left(\Delta^{2} U^{(k)}\right)^{T} .
$$

\subsection{Application of RRE}

The steps of application of the basic RRE technique are presented in table 1.

Table 1. RRE algorithm.

\begin{tabular}{|l|l|}
\hline Input & Vectors $\mathrm{u}^{(0)}, \mathrm{u}^{(1)}, \ldots, \mathrm{u}^{(\mathrm{k}+1)}$. \\
\hline Step 1 & Calculate $\Delta \mathrm{u}^{(\mathrm{i})}=\mathrm{u}^{(\mathrm{i}+1)}-\mathrm{u}^{(\mathrm{i})}$, for $i=0,1, \ldots, k$ \\
\hline & $\begin{array}{l}\text { Set } \Delta U^{(k+1)}=\left[\Delta u^{(0)}, \Delta u^{(1)}, \ldots, \Delta u^{(k)}\right] . \\
\text { Compute the } \mathrm{QR} \text { factorization of } \Delta U^{(k+1)}, \text { namely, } \Delta U^{(k+1)}=Q^{(\mathrm{k})} \mathrm{R}^{(\mathrm{k})} .\end{array}$ \\
\hline Step 2 & $\begin{array}{l}\text { Solve the linear system } \\
\left(R^{(k)}\right)^{T} R^{(k)} d^{(k)}=e ; \\
\text { where } d^{(k)}=\left[d_{0}^{(k)}, d_{1}^{(k)}, \ldots, d_{k}^{(k)}\right]^{T} \text { and } e=[1,1, \ldots, 1]^{T} . \\
\text { Set } \lambda=\left(\sum_{i=0}^{k} d_{i}^{(k)}\right)^{-1}, \lambda \in \mathbb{R}^{+} . \\
\text {Set } \beta_{i}^{(k)}=\lambda d_{i}^{(k)}, \text { for } \mathrm{i}=0,1, \ldots, \mathrm{k} .\end{array}$ \\
\hline Step 3 & $\begin{array}{l}\text { Compute } \xi^{(k)}=\left[\xi_{0}^{(k)}, \xi_{1}^{(k)}, \ldots, \xi_{k-1}^{(k)}\right]^{T}, \\
\text { where } \xi_{0}^{(k)}=1-\beta_{0}^{(k)} \text { and } \xi_{j}^{(k)}=\xi_{j-1}^{(k)}-\beta_{j}^{(k)}, 1 \leq \mathrm{j} \leq \mathrm{k}-1 . \\
\text { Compute } s^{(k)}=u^{(0)}+Q^{(k-1)}\left(R^{(k-1)} \xi^{(k)}\right) .\end{array}$ \\
\hline
\end{tabular}

When RRE is applied, as $\mathrm{k}$ increases this technique becomes increasingly expensive because of the work requirement which grows quadratically. Also, the storage requirement grows linearly with $\mathrm{k}$. Then, RRE should be restarted periodically every a number of steps $r$ where $r$ is an integer greater than 1. The choice of $r$ is analyzed in [8] and it has been seen that a small integer $r$ is sufficient. 
Here, three different ways of applying the restarted RRE to iteration (3) are listed in Tables 2,3 and 4. First, the restarted RRE is applied to the vector sequence $\left\{\mathrm{w}^{(\mathrm{k})}\right\}_{\mathrm{k} \in \mathrm{N}}$. Then, it is applied to the vector sequences $\left\{\mathrm{u}^{(\mathrm{k})}\right\}_{\mathrm{k} \in \mathrm{N}}$ and $\left\{\mathrm{v}^{(\mathrm{k})}\right\}_{\mathrm{k} \in \mathrm{N}}$ separately. Finally, it is applied only on $\left\{\mathrm{v}^{(\mathrm{k})}\right\}_{\mathrm{k} \in \mathrm{N}}$ to get a better approximation $\mathrm{s}^{(\mathrm{k})}$, then compute $\mathrm{u}^{(\mathrm{k})}$ using the following equation

$$
\mathrm{u}^{(\mathrm{k})}=\left(I_{n}-\operatorname{diag}\left(\mathrm{P} s^{(k)}\right)\right)^{-1} \mathrm{e}
$$

Table 2. Application of restarted RRE to $\left\{\mathrm{w}^{(\mathrm{k})}\right\}$.

\begin{tabular}{|c|l|}
\hline Step 1 & For $k=0, u^{(0)}=v^{(0)}=0$. Set $\mathrm{r}=4$. Then, $w^{(0)}=0$. \\
\hline Step 2 & $\begin{array}{l}\text { For } k=1,2, \ldots \\
\text { For } j=0,1, \ldots, r-1 \\
g^{(0)}=u^{(k-1)} ; h^{(0)}=v^{(k-1)} ; s^{(0)}=\left(g^{(0)}, h^{(0)}\right)^{T} ; \\
g^{(j+1)}=\left(I_{n}-\operatorname{diag}\left(\mathrm{P} h^{(j)}\right)\right)^{-1} \mathrm{e} ; \\
h^{(j+1)}=\left(I_{n}-\operatorname{diag}\left(\mathrm{Q} g^{(j+1)}\right)\right)^{-1} \mathrm{e} ; \\
s^{(j+1)}=\left(g^{(j+1)}, h^{(j+1)}\right)^{T} .\end{array}$ \\
\hline Step 3 & $\begin{array}{l}\text { Calculate the approximations } t^{(r-1)} \text { via applying the RRE } \\
\text { algorithm of table } 1 \text { on the vectors }\left(s^{(0)}, s^{(1)}, \ldots, s^{(r)}\right) .\end{array}$ \\
\hline Step 4 & If $t^{(r-1)}$ satisfies accuracy test, stop. Else, $w^{(k)}=t^{(r-1)}$. \\
\hline
\end{tabular}

Table 3. Application of restarted RRE to $\left\{\mathrm{u}^{(\mathrm{k})}\right\}$ and $\left\{\mathrm{v}^{(\mathrm{k})}\right\}$.

\begin{tabular}{|c|l|}
\hline Step 1 & For $k=0, u^{(0)}=v^{(0)}=0$. Set $\mathrm{r}=4$. \\
\hline Step 2 & $\begin{array}{l}\text { For } k=1,2, \ldots \\
\text { For } j=0,1, \ldots, r-1 \\
g^{(0)}=u^{(k-1)} ; h^{(0)}=v^{(k-1)} ; \\
g^{(j+1)}=\left(I_{n}-\operatorname{diag}\left(\mathrm{P} h^{(j)}\right)\right)^{-1} \mathrm{e} ; \\
h^{(j+1)}=\left(I_{n}-\operatorname{diag}\left(\mathrm{Q} g^{(j+1)}\right)\right)^{-1} \mathrm{e} ;\end{array}$ \\
\hline Step 3 & $\begin{array}{l}\text { Calculate the approximations } t_{1}^{(r-1)} \text { and } t_{2}^{(r-1)} \text { via applying } \\
\text { the RRE algorithm of table } 1 \text { on the vectors } \\
{\left[\left(g^{(0)}, h^{(0)}\right)^{T}, \ldots,\left(g^{(r)}, h^{(r)}\right)^{T}\right]}\end{array}$ \\
\hline Step 4 & $\begin{array}{l}\text { If } t_{1}^{(r-1)} \text { and } t_{2}^{(r-1)} \text { satisfies accuracy test, stop. } \\
\text { Else, } u^{(k)}=t_{1}^{(r-1)} \text { and } v^{(k)}=t_{2}^{(r-1)} .\end{array}$ \\
\hline
\end{tabular}


Now, we will apply the restarted RRE on $\left\{\mathrm{v}^{(\mathrm{k})}\right\}_{\mathrm{k} \in \mathrm{N}}$ only. Suppose that for all $\mathrm{t} \in \mathbb{R}^{\mathrm{n}}, \mathrm{K}$ and $\mathrm{L} \in \mathbb{R}^{\mathrm{nxn}}$, there exist two functions $f_{K}$ and $f_{K, L}$ in $\mathbb{R}^{\mathrm{n}}$ defined by

$$
f_{K}(t)=\left(I_{n}-\operatorname{diag}(\mathrm{Kt})\right)^{-1} \mathrm{e} \quad \text { and } f_{K, L}(t)=f_{K}\left(f_{L}(t)\right) .
$$

Equations (5) will be used in implementing the final application presented in Table 4.

Table 4. Application of restarted RRE to $\left\{\mathrm{v}^{(\mathrm{k})}\right\}$.

\begin{tabular}{|l|l|}
\hline Step 1 & For $k=0, u^{(0)}=v^{(0)}=0$. Set $\mathrm{r}=4$. \\
\hline Step 2 & $\begin{array}{l}\text { For } k=1,2, \ldots \\
\text { For } j=0,1, \ldots, r-1 \\
\\
g^{(0)}=v^{(k-1)} ; ; \\
g^{(j+1)}=f_{Q, P}\left(v^{j}\right) ;\end{array}$ \\
\hline Step 3 & $\begin{array}{l}\text { Compute the approximations } t^{(r-1)} \text { by applying the RRE } \\
\text { algorithm of table } 1 \text { on the vectors }\left(g^{(0)}, g^{(1)}, \ldots, g^{(r)}\right) .\end{array}$ \\
\hline Step 4 & $\begin{array}{l}\text { If } t^{(r-1)} \text { satisfies accuracy test, stop. } \\
\text { Else, } v^{(0)}=t^{(r-1)} ;\end{array}$ \\
\hline Step 5 & $u^{(k)}=f_{P}\left(v^{(k)}\right)$. \\
\hline
\end{tabular}

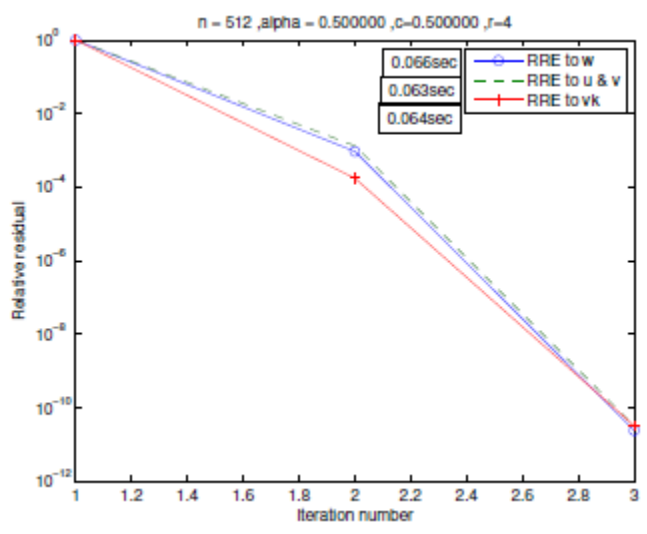

Fig. 1. Non critical case

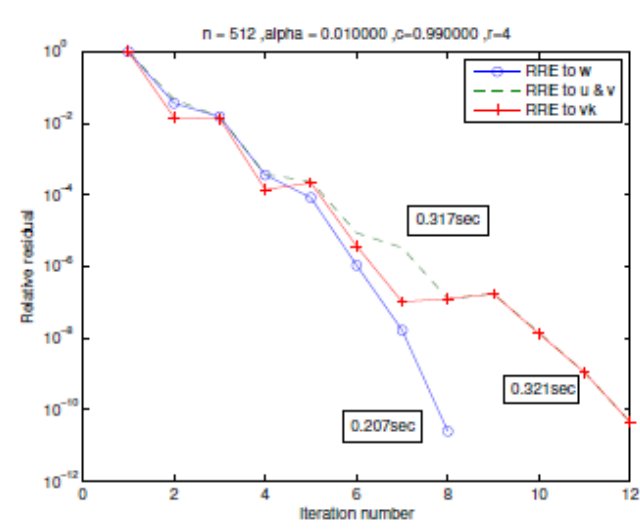

Fig. 2. Critical case

\section{Conclusion}

In this paper, different ways of application of the restarted RRE technique were presented. Figures 1 and 2 show the behaviour of the three applications both in the critical case, where the convergence rate is at its worst, and outside it. It has been seen that the application to 
$\left\{\mathrm{w}^{(\mathrm{k})}\right\}$ as in Table 2 was the best in the critical case. On the other hand, applying the restarted RRE only to $\left\{\mathrm{v}^{(\mathrm{k})}\right\}$ as in Table 4 worked the best in the non critical case.

\section{References}

1. J. Juang, Global existence and stability of solutions of matrix Riccatiequations, J. Math. Anal. Appl., 258(1), pp. 1-12 (2001)

2. J. Juang, W.W. Lin, Nonsymmetric algebraic Riccati equatioins and Hamiltonian-like matrices, SIAM J.Matrix Anal. Appl., 20, pp. 228-243 (1999)

3. V. Ramaswami, Matrix analytic methods for stochastic fluid flows, In D. Smith and P. Hey, editors, Teletraffic Engineering in a Competitive World, Proceedings of the 16th International Teletraffic Congress,Elsevier Science B.V., Edimburgh, UK, pp. 10191030 (1999)

4. L.C.G. Rogers, Fluid models in queueing theory and Weiner-Hopf factorization of Markov chains, Ann. Appl. Probab, 4(2), pp. 390-413 (1994)

5. D. Williams, A 'potential-theoretic'" note on the quadratic Weiner-Hopf equation for Q-matrices, In Seminar on Probability, XVI, 920, pp.91-94, Springer, Berlin (1982)

6. L.Z. Lu, Solution form and simple iteration of a nonsymmetric algebraic Riccati equation arising in transport theory, SIAM J. Matrix Anal. Appl., 26, pp. 679-685 (2005)

7. Y. Lin, A class of iterative methods for solving nonsymmetric algebraic Riccati equations arising in transport theory, Comput. Math. Appl., 56, pp. 3046-3051 (2008)

8. R. El-Moallem, H. Sadok, Vector extrapolation applied to algebraic Riccati equations arising in transport theory, ETNA (Electronic Transactions on Numerical Analysis Journal), volume 40, pp. 489-506 (2013)

9. R.P. Eddy, Extrapolating to the limit of a vector sequence., In: P.C.C. Wang, Editor, Information Linkage between Applied Mathematics and Industry, Academic Press, New York, pp. 387-396 (1979)

10. M. Mesina, Convergence acceleration for the iterative solution of the equations $X=A X+f$ ,Comput.Methods Appl. Mech. Engrg., 10, pp. 165-173 (1977) 